\title{
Efecto gradiente del calentamiento global
}

César Mauricio Urbizo Vivas *

\section{RESUMEN}

Este artículo presenta al lector un estudio sobre el calentamiento global bajo el enfoque de la termodinámica. Al aplicar las leyes de este enfoque se describe el mecanismo termodinámico del cambio climático. A partir de criterios físicoquímicos, se descubre el calor residual o de desecho como otro posible factor causante del aumento de la temperatura global. Lo anterior se ha denominado "Efecto Gradiente de Calor en la Atmósfera". Además, se explica la razón del por qué algunos gases contribuyen al calentamiento global. También se analiza la influencia que tienen las energías alternativas en este fenómeno.

Palabras clave: Efecto Gradiente de Calor, Calor Residual, Capacidad Calorífica, Máquina Térmica, Energía Interna

\section{ABSTRACT}

This article introduces the reader to a study of global warming under the framework of thermodynamics, applying laws it describes the thermodynamic mechanism of climate change. From physical-chemistry criteria, residual heat or waste is discovered as another possible factor in the rising global temperature, which has been termed "Gradient Effect of Heat in the Atmosphere". Explains the reason why some gases contribute to global warming and discusses the influence of alternative energy in this phenomenon.

Key words: Gradient Effect, Residual Heat, Heat Capacity, Thermal Machine, Internal Energy 


\section{INTRODUCCIÓN}

El calentamiento global es el aumento desmedido de la temperatura promedio de los gases que componen la atmósfera. En las últimas décadas este fenómeno atmosférico ha tomado considerable importancia debido a las proporciones mundiales. Por ser un factor influyente en la economía mundial y en todas las formas de vida que habitan en el planeta tierra, ha ocupado un espacio en las agendas de distintas asambleas de relevancia internacional.

Estudios previos han demostrado que la acumulación de gases aislantes en la atmósfera que impiden la liberación de energía en forma de calor desde la tierra hacia el resto del universo, provoca este aumento en la temperatura y es lo que se conoce como "efecto invernadero".

El artículo que a continuación se presenta, pretende exponer otra causa potencial y significativa de este fenómeno basado en las interacciones energéticas. Para lograr el objetivo se ubicó este comportamiento bajo el lente de la Termodinámica. Ésta utilizada como herramienta principal, da respuestas de por qué y cómo ocurre el calentamiento global.

\section{"EFECTO GRADIENTE DE CALOR EN LA ATMÓSFERA"}

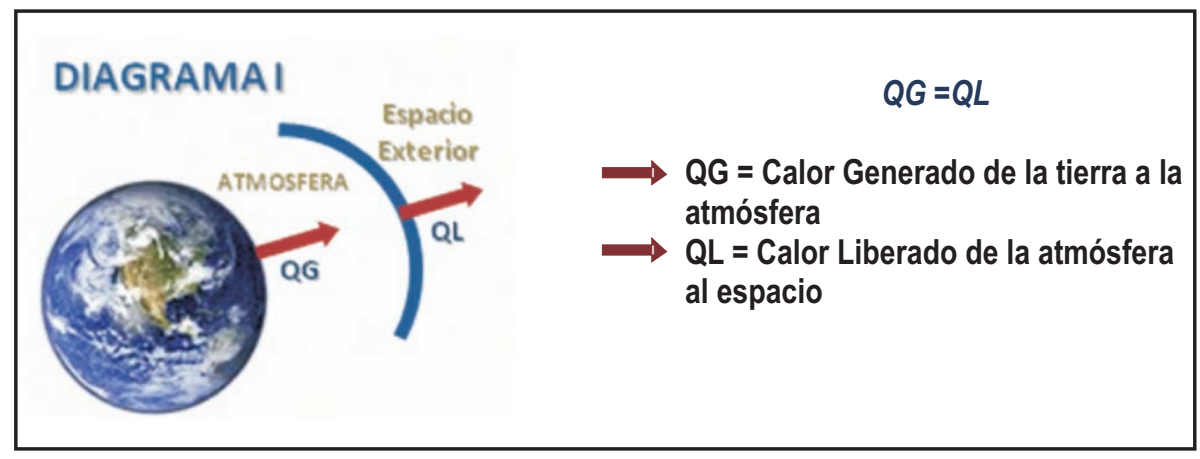

Si los gases de la capa atmosférica fuesen demasiado permeables, la producción de energía liberada sería alta con respecto a la cantidad de energía generada. Ésto traería como consecuencia un descenso en la temperatura; sumergido al planeta, con todo lo que habita en él en un mundo congelado donde sólo las criatura especializadas a los ambientes extremadamente fríos subsistirían; excluyendo probalemente a los humanos de esta privilegiada condición. 


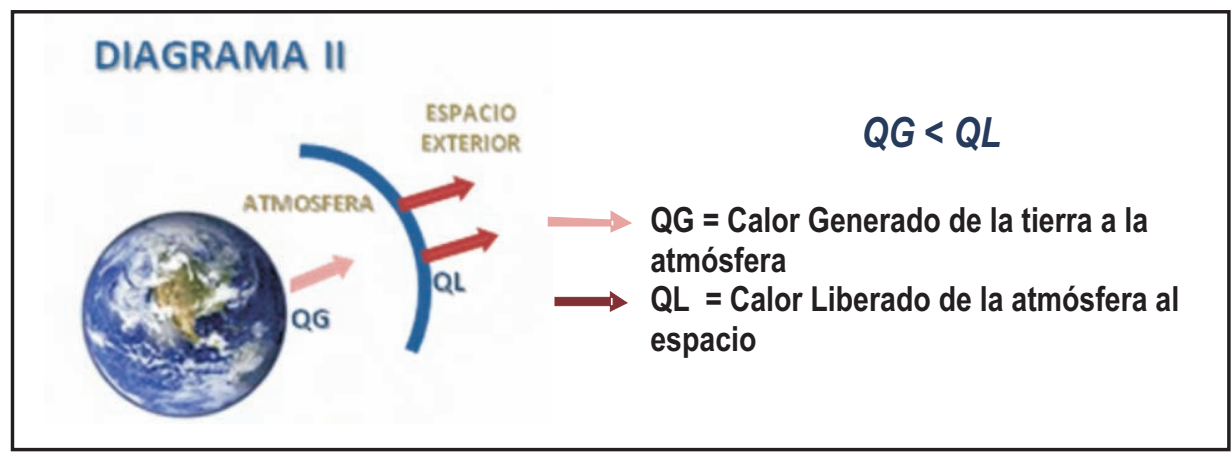

Por otra parte, el escenario contrario ocurre cuando la cantidad de energía calórica generada sobrepasa la fracción de energía liberada. Lo anterior causa una retención del calor que aumenta con el tiempo y que provoca un aumento gradual de la temperatura junto a consecuencias letales a largo plazo. A ésto se le conocerá como "Efecto Gradiente de Calor en la Atmósfera". El efecto gradiente de calor sumado a la acumulación de gases aislantes conocido como efecto invernadero, convierten el futuro en algo incierto y poco prometedor para todas las especies que habitan el planeta.

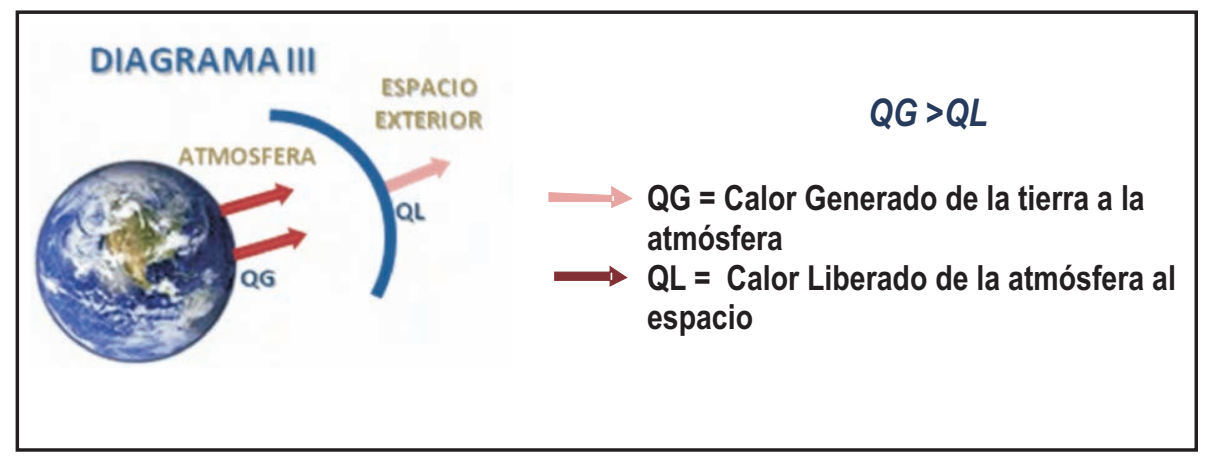

\section{TERMODINÁMICA}

La termodinámica es la rama de la físico-química cuya misión es el estudio de las causas y mecanismos de los distintos intercambios de energía que ocurren en la naturaleza, además de la cuantificación del trabajo realizado por y sobre el sistema y la energía absorbida y liberada por éste. La termodinámica se rige por leyes:

\section{Ley Cero de la Termodinámica}

Esta ley también es conocida como "Ley del Equilibrio Térmico" . "si un cuerpo C 
inicialmente está en equilibrio térmico con un cuerpo $A y$ con un cuerpo $B$, entonces $A$ y B también están en equilibrio térmico entre sí". En el universo toda materia que interactúa energéticamente con otra a diferente temperatura, tendrá la tendencia de alcanzar la misma temperatura final o de equilibrio con la otra.

Ésto se conoce como "Equilibrio Térmico". Existen tres mecanismos para alcanzar el equilibrio térmico:

1. Conducción: La energía fluye en forma de calor entre dos o más cuerpos que están en contacto directo a distinta temperatura. Por ejemplo: una olla de metal fría colocada sobre una hornilla caliente de una estufa.

2. Radiación: La energía se transfiere a través de una radiación electromagnética. Por ejemplo: la luz del sol cuando calienta la atmósfera.

3. Convección: El traspaso de energía ocurre por el desplazamiento de la masa de un fluido de un lugar a otro. Por ejemplo: los acondicionadores de aire.

\section{Primera Ley de la Termodinámica}

Se conoce también con el nombre de "Ley de la Conservación de la Energía". La energía en el universo no puede ser creada ni ser destruida, sólo transformada de una forma a otra. El universo considerado como un sistema aislado, o sea que no absorbe ni libera energía con su entorno, la energía en él es constante.

La primera ley de la termodinámica puede ser definida matemáticamente como:

$$
\mathrm{U}=\mathbf{Q}-\mathbf{W}
$$

\section{$\mathrm{U}=$ Energía Interna \\ $Q=$ Calor \\ $\mathbf{W}=$ Trabajo}

\section{MÁQUINA TÉRMICA}

La máquina térmica es un aparato idealizado que absorbe calor a partir de una fuente; parte es utilizado para efectuar un trabajo, y el resto para liberar calor. Por ser este calor un producto de desecho se le denominará en adelante "Calor Residual".

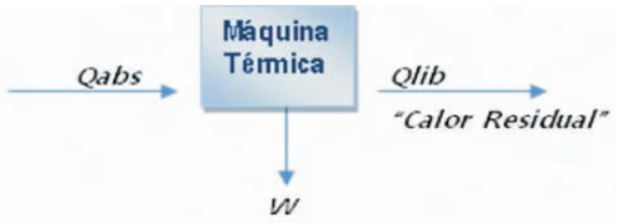

Qabs $=$ Calor absorbido

Qlib =Calor liberado 
La cantidad de trabajo que realiza la máquina depende directamente de la cantidad de calor que absorbe y de la eficiencia de la máquina. El trabajo realizado no puede ser mayor que la cantidad de calor absorbido:

\section{$W<Q$ abs}

Como el cambio de energía interna $(\Delta U)$ es la diferencia entre el calor absorbido y el respectivo trabajo realizado por la máquina térmica, da como resultado valores positivos:

\section{$\Delta \mathrm{U}=$ positivo (+)}

Para los gases ideales el cambio de energía interna es igual al cambio en la temperatura multiplicado por su correspondiente constante de proporcionalidad:

\section{$\Delta U=K \Delta T$}

$$
\begin{aligned}
& \text { ? T= cambio de temperatura } \\
& \mathrm{K}=\text { constante de proporcionalidad }
\end{aligned}
$$

Existe una relación directamente proporcional entre el cambio de energía interna y el cambio de temperatura:

\section{$\Delta U \propto \Delta T$}

Como se explicó anteriormente, el cambio de energía interna es positivo y debido a su relación directa con el cambio de temperatura ésta da cambios positivos:

\section{$\Delta \mathrm{U}=$ positivo (+)}

Lo que se interpreta como un aumento en la temperatura de los gases.

\section{GASES DE EFECTOINVERNADERO}

¿Cuáles son los gases que producen calentamiento global y por qué? La respuesta a esta pregunta se encuentra en la constante de proporcionalidad $\boldsymbol{K}$.

La $\boldsymbol{K}$ en este caso recibe el nombre de capacidad calorífica molar a volumen constante (Cv) y sus unidades en el sistema internacional (SI) son: 


\section{$\frac{J}{m o l-k}$}

Estas unidades nos indican cuánta energía en Joules (J) es necesaria para aumentar en una unidad de temperatura (Kelvin "k") una masa de un mol de gas. Una partícula de gas monoatómica posee 3 grados de libertad siendo éstos los vectores sobre los ejes que componen el plano tridimensional (X, YyZ).

Según la mecánica estadística y bajo criterios matemáticos del modelo cinético molecular de los gases ideales, la capacidad calorífica molar a volumen constante está definida de la siguiente manera:

$$
\begin{array}{r}
C v=3\left(\frac{1}{2} k T\right) \quad \begin{array}{r}
\mathrm{K}=\text { la constante de proporcionalidad } \\
\mathrm{T}=\text { Temperatura }
\end{array} \\
\text { El } 3 \text { denota los grados de libertad } \\
C v=\left(\frac{3}{2} R\right) \quad \mathrm{R}=\text { constante de proporcionalidad de los gases ideales. } \\
\text { Para Sl el valor es } \mathbf{8 . 3 1 4} \mathrm{J} / \mathbf{m o l}-\mathbf{k}
\end{array}
$$

Los gases diatómicos presentan dos grados adicionales de libertad además de los 3 originales, corresponden a ejes de rotaciones ortogonales entre sí y al eje de la molécula. Todo ésto se traduce como una mayor capacidad calorífica molar:

$$
C v=\left(\frac{5}{2} R\right)
$$

Para moléculas diatómicas a mayores cantidades de energía absorbida, se provoca vibración en los enlaces de la molécula, ésto le confiere otros 2 grados adicionales de libertad haciendo un total de 7:

$$
C v=\left(\frac{7}{2} R\right)
$$

Una molécula de gas conformado por 30 más átomos se conoce como "poliatómico". Estas moléculas poseen 6 grados de libertad, 3 grados de libertad traslacional y 3 grados de libertad rotacional, siempre y cuando los átomos no estén 
dispuestos en línea recta (como el $\mathrm{CO}_{2}$ ).

$$
C v=3 R
$$

Los grados vibratorios para gases poliatómicos sólo se alcanzan a temperaturas sumamente altas y pueden ser mayores que dos.

Tabla No. 1

\begin{tabular}{|l|c|c|}
\hline \multicolumn{1}{|c|}{ Tipo de Gas } & Gas & Cv (J/mol-K) \\
\hline Monoatómicos & $\mathrm{He}$ & 12.47 \\
\hline Diatómico & $\mathrm{Ar}$ & 12.47 \\
& $\mathrm{H}_{2}$ & 20.42 \\
& $\mathrm{~N}_{2}$ & 20.76 \\
\hline & $\mathrm{O}_{2}$ & 21.10 \\
\hline Poliatómicos & $\mathrm{CO}$ & 20.85 \\
\hline & $\mathrm{CO}_{2}$ & 28.46 \\
\hline & $\mathrm{SO}_{2}$ & 31.39 \\
\hline & $\mathrm{H}_{2} \mathrm{~S}$ & 25.95 \\
\hline & $\mathrm{H}_{2} \mathrm{O}$ & 35.33 \\
\hline
\end{tabular}

Para gases diatómicos, los valores teóricos calculados difieren poco de los detectados experimentalmente. Como es evidente los gases triatómicos poseen una capacidad calorífica superior. Ésto se traduce como un poder aislante mayor.

\section{EFICIENCIA}

La eficiencia de una máquina térmica es la tasa entre el trabajo realizado con respecto al calor absorbido. Ésta debe ser numéricamente menor que uno, recordando que el trabajo es una fracción del calor absorbido:

$$
\mathcal{E}=\frac{\mathrm{W}}{\text { Qabs }}<1 \quad \begin{array}{r}
\varepsilon=\text { Eficiencia } \\
\mathrm{W}=\text { Trabajo } \\
\mathrm{Q}=\text { Calor Absoluto }
\end{array}
$$

Para logar una eficiencia teórica máxima igual a uno, todo el calor absorbido debe ser transformado en trabajo y no liberar calor residual.

$$
\text { Qabs }=W \quad \Rightarrow \quad \frac{\mathrm{W}}{Q a b s}=1
$$




\section{COMBUSTIÓN}

La combustión es la reacción más antigua y conocida por el hombre y cuenta con un sin número de aplicaciones. Ocurre cuando un compuesto orgánico es sometido a altas temperaturas en presencia de un exceso de oxígeno.

\section{$\mathrm{C}_{\mathrm{n}} \mathrm{Hx}+\mathrm{O}_{2}$ (Exceso) $\longrightarrow \mathrm{nCO}+1 / 2 \mathrm{xH}_{2} \mathrm{O}+$ Calor $\quad \Delta \mathrm{H}=$ negativo(-) \\ Calor}

Es evidente en esta reacción la generación de dióxido de carbono y vapor de agua como gases de efecto invernadero. Debido a que el calor es un producto, permite clasificarla como una reacción "Exotérmica". La cantidad de energía liberada depende de la naturaleza de la sustancia que se quema y la podemos representar en función de una propiedad conocida como "Entalpía $(H)$ ".

Reacciones que desprenden calor se denotan con cambios de entalpía de signo negativo.

La gasolina posee un calor de combustión de 46,000 J/ g.

\section{MOTORES DE COMBUSTIÓN INTERNA}

Los motores de automóviles son un ejemplo típico de una maquina térmica. Éstos absorben energía del foco caliente, en este caso el combustible que arde ontroladamente en el interior del motor. De allí el nombre de combustión interna. Del calor de combustión producido por la reacción, una porción es utilizada y transformada en trabajo útil y la diferencia se libera a las fronteras del sistema como calor residual. Es por esa razón que los automóviles se calientan durante su uso y se enfrían al permanecer apagados.

\section{EFICIENCIADE MOTORES DE COMBUSTIÓN INTERNA}

Cada gramo de gasolina quemado produce $46,000 \mathrm{~J}$ de calor que son absorbidos por el motor. Un automóvil o un camión generan un promedio de 9,200 J de trabajo por ciclo:

$$
\varepsilon=\frac{\mathrm{W}}{Q a b s} \cdot 100
$$




$$
\begin{gathered}
\mathcal{E}=\frac{9200 \mathrm{~J}}{46000} \cdot 100 \\
\mathcal{E}=20 \%
\end{gathered}
$$

La eficiencia media de un automóvil o un camión es de 20\%, es lo mismo que decir un $80 \%$ de ineficiencia equivalente a calor residual.

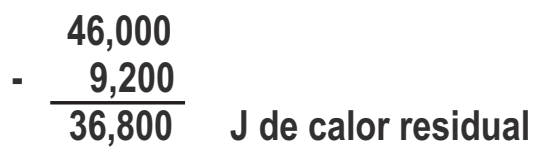

Por cada gramo de gasolina quemada, esta es la cantidad de calor liberado a la frontera del motor y al ambiente.

\section{ENTROPÍA DEL CALENTAMIENTO GLOBAL}

La segunda ley de la termodinámica posee varios enfoques. El primero define la orientación de los desplazamientos energéticos. El calor presenta un movimiento unidireccional, siempre de mayor a menor concentración calórica. Otro enfoque es una relación cuantitativa conocida como entropía. Un tercer punto de vista es en base al "desorden" o aleatoriedad del movimiento molecular.

En el calentamiento global se manifiesta la segunda ley de la termodinámica. El calor residual transmite energía a los gases atmosféricos y los calienta. Este fenómeno es natural, espontáneo e irreversible. La energía absorbida aumenta las velocidades moleculares de los gases; conduciendo a un crecimiento en la temperatura. Ésta a su vez incrementa la entropía y la aleatoriedad o desorden del sistema. Lo anterior demuestra el principio termodinámico que dice: todo fenómeno en la naturaleza va acompañado de un aumento en la entropía.

\section{LA TIERRA VISTA COMO UNA MÁQUINA TÉRMICA}

Toda máquina térmica necesita de una fuente de energía. La tierra cuenta con un suministro de energía casi inagotable que durante millones de años la ha alimentado. El sol, la estrella más cercana, es el proveedor y responsable de toda la actividad energética que se lleva a cabo en la tierra, incluso de la vida. El sol emana todo un caudal de energía radiante a través del sistema solar. Una pequeña fracción de ésta es absorbida por la tierra, según la ley cero de la termodinámica. 
De toda la energía atrapada, solamente un segmento es transformado en trabajo y utilizado para la subsistencia, crecimiento y desarrollo de la vida. La sección restante se libera a la atmósfera en forma de calor residual; basado en el primer principio de la termodinámica.

\section{ENERGÍAS ALTERNATIVAS}

Son aquellas que se obtienen a partir de fuentes de energía no convencionales. Por sus bajos costos de producción y precio razonablemente cómodo en el mercado, son una opción ante la constante demanda energética. En la actualidad existen diversas formas de energía alternativa, y se clasifican en función de la fuente de la cual se obtienen. Éstos manantiales deben ser abundantes, económicos y en algunos casos regenerables.

Los métodos de producción de algunas energías alternativas consisten en extraer energía de la fuente para calentar una masa de agua hasta su estado de vapor. Este gas es utilizado para impulsar una turbina que tiene acoplado un generador de energía.

No existe conversión de energía perfecta, donde no haya pérdidas en el transcurso del proceso, así que se desecha el residuo energético al ambiente. Como ejemplo está la Energía Térmica que obtiene el calor a partir de la reacción exotérmica de combustión de biomasa renovable, por ejemplo el desecho de la caña de azúcar. Propio de la naturaleza de esta reacción es la síntesis de gases de efecto invernadero y de calor residual como producto de desecho.

De igual manera la Energía Nuclear a través de una fisión nuclear produce inmensas cantidades de calor; liberando parte de éste al ambiente. Son productos secundarios para esta reacción, sustancias radioactivas de desecho y vapor de agua como gas de invernadero que escapa por las torres de enfriamiento. La Energía Geotérmica se obtiene extrayendo calor del manto terrestre. Con el calor residual que desprende, calienta las fuentes de agua utilizadas como refrigerante durante el proceso y no genera gases de invernadero.

Otros métodos para la obtención de energía no requieren calentar agua para transformarla en vapor, se basan en la conversión de energía mecánica a eléctrica, a partir de sustancias que se encuentran en la naturaleza como fluidos. La Energía Hidroeléctrica aprovecha la energía potencial y cinética del agua para imprimir impulso a las turbinas generadoras; de manera que no libera gases de efecto invernadero. 
Energía Eólica, su nombre procede del dios del viento de la mitología griega Eolo. La energía eólica proviene de la conversión de energía que se encuentra en las corrientes de aire que empujan las hélices de las turbinas que a su vez transmiten el movimiento a los generadores.

Las eficiencias de estas 2 últimas formas se basan en la relación que hay entre el potencial de entrada y de salida; dependiendo principalmente de la hidrodinámica y la aerodinámica de su diseño respectivamente.

Tabla No. 2

La tabla que a continuación se presenta, muestra los porcentajes promediados de eficiencias de algunas máquinas térmicas.

\begin{tabular}{|c|c|}
\hline Máquina & $\begin{array}{c}\text { Porcentaje de } \\
\text { Eficiencia }\end{array}$ \\
\hline Motor de Gasolina & $20 \%$ \\
\hline Motor Diesel & $35 \%$ \\
\hline Central Hidroeléctrica & $85 \%$ \\
\hline Central Eólica & $60 \%$ \\
\hline Central Térmica & $38 \%$ \\
\hline
\end{tabular}

\section{CONCLUSIONES}

- Al tomar en cuenta que es termodinámicamente imposible obtener eficiencias de $100 \%$, y basados en datos experimentales que demuestran la baja eficiencia de las máquinas térmicas, se considera la posibilidad de que la magnitud de calor residual generado hacia la atmósfera puede superar la magnitud de calor que la atmósfera libera hacia las fronteras del planeta; causando un efecto gradiente de calor.

- Los gases poliatómicos, especialmente los triatómicos desprendidos en las emisiones gaseosas, principalmente de procesos creados por la acción del hombre, contribuyen al calentamiento global debido a que poseen una capacidad calorífica alta; razón por la cual su poder aislante aumenta. 
- Las energías alternativas no son completamente limpias. Resuelven a corto plazo la necesidad de consumo energético pero su transformación implica la liberación de calor residual que se acumula significativamente a largo plazo; contribuyendo al efecto gradiente del calor.

- Las centrales hidroeléctricas y eólicas son las que menos efecto gradiente provocan, debido a sus porcentajes de eficiencia relativamente altos.

\section{AGRADECIMIENTOS}

El presente documento no pudo haberse realizado sin la gentil colaboración de las siguientes personas; quienes brindaron su invaluable apoyo, confianza e inspiración:

César Darío Urbizo, Dra. Marilyn Guevara Peña, Lic. Abril Urbizo Vivas, a mis padres Dr. Oscar A. Urbizo Solís, Dra. Marta O. Vivas Oviedo y principalmente a Dios.

Agradezco infinitamente el privilegio de haber gozado de la acertada asesoría y asistencia técnica de los siguientes profesionales distinguidos:

Lic. Itzel Moreno, Ing. Lizzeth Guevara, Luis Rivera M.Sc., Ing. Pablo Manzanares

\section{BIBLIOGRAFÍA}

Maron, Samuel H. y Prutton, Carl F. Fundamentos de Fisicoquímica. Última Edición. México: Limusa; 2006.

Resnick, Robert, Halliday, David y Krane Kenneth S. Física. Vol.1. Cuarta Edición. México: Cecsa; 1992.

Sears, Francis W., Zemansky, Mark W. Young, Hugh D. y Freedman, Roger A. Física Universitaria. Vol. 1. Undécima Edición. México: Pearson; 2004.

http://www.todomotores.cl/mecanica/eficiencia_termica.htm http://circuitoabierto.blogspot.com/2006/11/tema-2-cmo-funciona-un-motordiesel.html

http://books.google.hn/books?id=1rlBBXQhmCwC\&pg=PA68\&lpg=PA68\&dq =porcentaje+de+eficiencia+de+centrales+hidroel\%C3\%A9ctricas\&sourc e=bl\&ots=ihXZyb1j6i\&sig=cn1miVCcrM9JFSLMCI3bFWBBdw\&hl=es\&ei= XIKqTNfnJcG88gbNyPD_DA\&sa=X\&oi=book_result\&ct=result\&resnum=

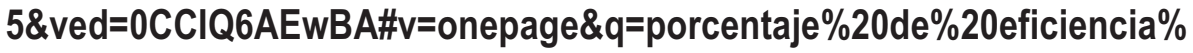
$20 \mathrm{de} \% 20$ centrales $\% 20$ hidroel $\%$ C $3 \%$ A9ctricas \&f=false http://www.energyadvocate.com/fw91.htm 